\title{
A NUMERICAL STUDY OF COUPLED NON-LINEAR EQUATIONS OF THERMO-VISCOUS FLUID FLOW IN CYLINDRICAL GEOMETRY
}

\author{
N. POTHANNA and P. APARNA \\ Department of Mathematics \\ VNR Vignana Jyothi Istitute of Engineering and Technology \\ Kukatpally, Hyderabad - 500090, INDIA \\ E-mail: pothareddy81@gmail.com; aparnapodila@gmail.com \\ R.S.R. GORLA* \\ Department of Mechanical Engineering \\ Cleveland State University \\ Cleveland, Ohio 44115 USA \\ E-mail: r.gorla@csuohio.edu
}

\begin{abstract}
In this paper we present numerical solutions to coupled non-linear governing equations of thermo-viscous fluid flow in cylindrical geometry using MATHEMATICA software solver. The numerical results are presented in terms of velocity, temperature and pressure distribution for various values of the material parameters such as the thermo-mechanical stress coefficient, thermal conductivity coefficient, Reiner Rivlin cross viscosity coefficient and the Prandtl number in the form of tables and graphs. Also, the solutions to governing equations for slow steady motion of a fluid have been obtained numerically and compared with the existing analytical results and are found to be in excellent agreement. The results of the present study will hopefully enable a better understanding applications of the flow under consideration.
\end{abstract}

Key words: Reiner Rivlin Cross viscosity, thermal conductivity, thermo-stress viscosity, Prandtl number.

\section{Introduction}

\subsection{Literature}

The basic equations of classical hydrodynamics are non-linear in the velocities and only a few instances have been reported in literature in which exact solutions could be obtained. The same situation applies to a higher extent in the case of non-Newtonian fluids, since even the constitutive relations qualifying these fluids are non-linear. The failure of the linear theories in predicting to a reasonable extent the mechanical behaviour of materials such as liquid polymers, fluid plastic, molten metals etc., subjected to stresses has been the motivating force behind the study of the non-linear theories for material description. The non-Newtonian nature of materials has been the subject of an extensive study for over one and half centuries. It is only in the last seven or eight decades that serious attempts have been made to extend these investigation in the realm of non-linearity. With the growing importance of non-Newtonian fluids in modern technology and industries, the investigations on such fluids are desirable. The non-linear theory reflecting the interaction/interrelation between thermal and viscous effects has been preliminarily studied by Koh and Eringen [5]. A systematic rational approach for such class of fluids has been developed by Green and Nagdhi $[2,3]$. Kelly[4] examined some simple shear flows of second order thermo-viscous fluids.

\footnotetext{
${ }^{*}$ To whom correspondence should be addressed
} 
The problem of a steady flow of a second order thermo- viscous fluid over an infinite plate was studied by Rao and Ramacharyulu [11]. The steady flow of a thermo-viscous fluid through straight tubes was examined by Rao and Ramacharyulu [12]. In this paper, the velocity and temperature fields have been obtained by employing a four step recursive approach. A steady slow motion through a circular tube was studied by Rao and Ramacharyulu [13]. The effects of large and small values of the thermo physical parameters on the flow field have been discussed in this paper. Rao and Ramacharyulu [10] examined some steady state problems dealing with certain flows of thermo-viscous fluids. Anuradha [1] and Nagaratnam [9] studied the flow in plane, cylindrical and spherical geometries. Recently, Pothanna et al. [18] et al studied unsteady forced oscillations of a fluid bounded by a rigid bottom. Pothanna et al. [15, 16, 17] also investigated certain flows of thermo-viscous fluids in a porous slab bounded between two horizontal parallel plates and the effects of various physical parameters on the flow field have been discussed. Srinivas et al. [19] studied a slow steady motion of a thermo-viscous fluid between two parallel plates with constant pressure and temperature gradients.

Curved pipe/annular configurations are of immense practical importance in almost all piping systems, the human cardiovascular system and in several engineering devices such as heat and mass exchanges, chemical reactors, chromatography columns and other processing equipment. Owing to the wide range of applications, the interest in the study of flow characteristics in these configurations has grown enormously during the last decades. Green and Nagdhi [3] has given a new thermo-viscous theory for fluids. Green and Nagdhi [2] explained a dynamical theory of interacting continua. Longlois [6] examined a slow steady flow of viscoelastic fluids through non-linear tubes. Muthuraj and Srinivas [7] studied the flow of a Thermo-viscous fluid through an annular tube with constriction. The motion is assumed to be slow and the governing equations have been solved in terms of the modified Bessel functions. Srinivas and Muthuraj [20] also examined the flow of a thermo-viscous fluid in a radially non-symmetric constricted tube. Srinivas et al. [21], studied peristaltic transport of a thermo-viscous fluid. Hossain et al. [8], studied a fluctuating flow of a thermo-micropolar fluid past a vertical surface. Anuradha et al. [1] investigated the problem of a steady flow of a thermo-viscous fluid through a moving circular pipe .

It can be noted, from the past studies, that the governing equations of the fluid flow in cylindrical geometry are linear in nature and the closed form solutions are obtained using various analytical methods. However, in reality the flow and heat transfer problems are non-linear in nature. The basic governing equations of the present study are coupled and highly non linear in nature. Hence, it is important to study the solutions of non-dimensional non-linear partial differential equations which govern the momentum and energy. They are solved numerically with the help of MATHEMATICA software solvers. As far as known, this problem has not been discussed in the literature.

\subsection{Basic equations} equations.

The flow of an incompressible homogeneous thermo-viscous fluid satisfies the usual conservation

The equation of continuity(law of conservation of mass)

$$
v_{i, i}=0 .
$$

The equation of momentum(law of conservation of momentum)

$$
\rho\left[\frac{\partial v_{i}}{\partial t}+v_{k} v_{i, k}\right]=\rho f_{i}+t_{j i, j},
$$

and the energy equation(law of conservation of energy)

$$
\rho c \dot{\eta}=t_{i j} d_{i j}-q_{i, i}+\rho \gamma
$$


where

$f_{i}=i^{\text {th }}$ component of external force per unit mass

$c=$ specific heat

$\rho=$ density of the fluid

$\gamma=$ energy source per unit mass

$q_{i}=i^{\text {th }}$ component of heat flux bivector $=\epsilon_{i j k} h_{j k} / 2$

$d_{i j}=\left(u_{i, j}+u_{j, i}\right) / 2=$ rate of deformation tensor

$b_{i j}=\epsilon_{i j k} \eta_{k}=$ thermal gradient bivector

where $u_{i}$ is the $i^{\text {th }}$ component of velocity and $\eta$ is the temperature field.

As proposed by Koh and Eringen [5], the constitutive equations for the stress tensor and heat flux bivector of second order thermo-viscous fluids coupled in $d$ and $b$ are given by

and

$$
t=\alpha_{1} I+\alpha_{3} d+\alpha_{5} d^{2}+\alpha_{6} b^{2}+\alpha_{8}(d b-b d)
$$

$$
h=\beta_{1} b+\beta_{3}(b d+d b)
$$

where the coefficients $\alpha_{i}^{\prime s}$ and $\beta_{i}^{\prime s}$ are scalar polynomials in $t r d, t r d^{2}, t r b^{2}$. Explicit expressions for the constitutive coefficients $\alpha_{i}^{\prime s}$ and $\beta_{i}^{\prime s}$ for the second order theory may be obtained as

$$
\begin{aligned}
& \alpha_{1}=\alpha_{1000}+\alpha_{1010} t r d+\alpha_{1020} \operatorname{tr} d^{2}+\alpha_{1002} \operatorname{tr} b^{2}, \\
& \alpha_{3}=\alpha_{3010}+\alpha_{3020} \operatorname{tr} d \\
& \alpha_{5}=\alpha_{5020}, \\
& \alpha_{6}=\alpha_{6002}, \\
& \alpha_{8}=\alpha_{8011} \\
& \beta_{1}=\beta_{1001}+\beta_{1011} t r d
\end{aligned}
$$

and

$$
\beta_{3}=\beta_{1011},
$$

the secondary coefficients $\alpha_{i s r t}$. and $\beta_{i s r t}$ are functions of $\rho$ and $\eta$.

The fluids defined by the constitutive Eqs (1.4) and (1.5) with constant values for the constitutive coefficients $\alpha_{i}^{\prime s}$ and $\beta_{i}^{\prime s}$ may be called second order thermo-viscous fluids. This is the simplest model of a thermo-viscous fluid which exhibits an interaction between mechanical and thermal phenomena.

\section{Mathematical analysis}

Consider the steady flow of a second order thermo-viscous fluid around a circular pipe moving with a given velocity (Fig.1). Introducing the cylindrical polar coordinate system $(r, \theta, z)$ where the $z$-axis is along 
the axis of the pipe and $r$ is the radial distance from the centre of the circular pipe. The flow is represented by the velocity $(0,0, w(r))$ and the temperature $\eta(r)$. This choice of velocity evidently satisfies the continuity equation.

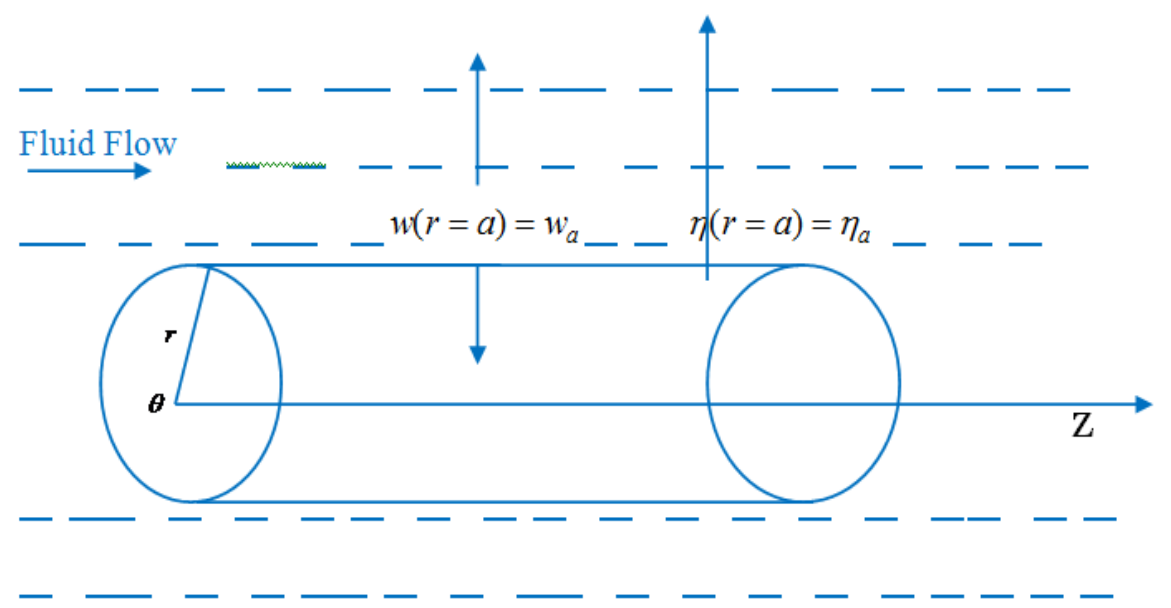

Fig.1. Schematic representation of fluid flow.

The flow is assumed to be under the action of constant temperature and pressure gradients. It is also assumed that the pipe is moving with a constant velocity $w_{a}$ and $\eta_{a}$ is the temperature prescribed on the boundary. Under these assumptions the basic equations characterizing the flow are the following:

In the radial direction

$$
\frac{\partial p}{\partial r}=\mu_{c}\left\{\frac{1}{r}\left(\frac{\partial w}{\partial r}\right)^{2}+2 \frac{\partial w}{\partial r} \frac{\partial^{2} w}{\partial r^{2}}\right\}-\alpha_{6} \frac{1}{r}\left(\frac{\partial \eta}{\partial r}\right)^{2}+\rho F_{r}
$$

In the transverse direction

$$
\rho F_{\theta}=\alpha_{8}\left\{\frac{\partial w}{\partial r} \nabla^{2} \eta+\frac{\partial \eta}{\partial r} \nabla^{2} w\right\}
$$

In the axial direction (z-direction)

$$
\frac{\partial p}{\partial z}=\mu \nabla^{2} u-\alpha_{6} c_{2} \nabla^{2} \eta+\rho F_{z}
$$

and the energy equation

$$
\rho c u\left(\frac{\partial \eta}{\partial z}\right)=\mu \frac{1}{r}\left(\frac{\partial w}{\partial r}\right)^{2}-\alpha_{6}\left(\frac{\partial \eta}{\partial z}\right)\left(\frac{\partial w}{\partial r}\right)\left(\frac{\partial \eta}{\partial r}\right)+k \nabla^{2} \eta+\beta_{3}\left(\frac{\partial \eta}{\partial z}\right) \nabla^{2} u .
$$

The appropriate boundary conditions of the problem are 


$$
\begin{array}{ll}
w(r=a)=w_{a}, & w(r=\infty)=\text { finite }, \quad p(r=a)=p_{a}, \\
\eta(r=a)=\eta_{a}, \quad \eta(r=\infty)=\text { finite }
\end{array}
$$

where $w_{a}, p_{a}$ and $\eta_{a}$ are respectively the constant velocity, pressure and temperature prescribed on the boundary.

Further, it is assumed that the flow is generated with the constant pressure gradient $\frac{\partial p}{\partial z}\left(=-C_{1}\right)$ and the temperature gradient $\frac{\partial \eta}{\partial z}\left(=C_{2}\right) . \rho\left(F_{r}, F_{\theta}, F_{z}\right)$ are the components of the external force/volume. It is assumed that there is no external force down the tube length, i.e., $F_{r}, F_{\theta}$ and $F_{z}$ are zero.

Introducing the following non-dimensional quantities

$$
\begin{aligned}
& r=a R, \quad w=\frac{a^{2}}{\mu} W, \quad p=\frac{a^{2}}{\mu^{2}} P, \quad \eta=\eta_{a} T, \quad a_{6}=\frac{\alpha_{6} \eta_{a}}{a^{2}}, \quad b_{3}=\frac{\beta_{3}}{\mu}, \\
& m_{1}=\frac{\mu^{4} \eta_{a}}{a^{2}}, \quad m=\frac{\eta_{a} c}{p_{r}} \text { and } p_{r}=\frac{\mu c}{k} \text { (Prandtl number). }
\end{aligned}
$$

Using the above non-dimensional quantities, the equations of momentum in the radial and axial direction and the energy equation in non-dimensional form now reduce to:

In the radial direction

$$
\frac{\partial P}{\partial R}=\mu_{c}\left\{\frac{1}{R}\left(\frac{\partial W}{\partial R}\right)^{2}+2 \frac{\partial W}{\partial R} \frac{\partial^{2} W}{\partial R^{2}}\right\}-m_{1} a_{6} \frac{1}{R}\left(\frac{\partial T}{\partial R}\right)^{2}
$$

In the axial direction

$$
-C_{1}=\mu \nabla^{2} W-a_{6} C_{2} \nabla^{2} T
$$

and the energy equation

$$
\rho c C_{2} W=\mu \frac{1}{R}\left(\frac{\partial W}{\partial R}\right)^{2}-a_{6} C_{2}\left(\frac{\partial W}{\partial R}\right)\left(\frac{\partial T}{\partial R}\right)+k \nabla^{2} T+b_{3} C_{2} \nabla^{2} W
$$

where $\nabla^{2}=\frac{\partial^{2}}{\partial R^{2}}+\frac{1}{R} \frac{\partial}{\partial R}$ and $a_{6}$ is the non-dimensional thermo-mechanical stress viscosity coefficient and $b_{3}$ is the non-dimensional strain thermal conductivity coefficient.

The boundary conditions of the problem reduce to

$$
\begin{aligned}
& W(R=1)=W_{a}, \quad W(R=\infty)=\text { finite, } \quad P(R=1)=P_{a}, \\
& T(R=l)=1 \text { and } T(R=\infty)=\text { finite. }
\end{aligned}
$$




\section{Numerical study of the problem}

The governing coupled highly non-linear differential Eqs (2.7)-(2.9) together with the boundary conditions (2.10) for the velocity, temperature and pressure distributions are solved by using the R-K method of $6^{\text {th }}$ order with shooting methods with the help of MATHEMATICA software ND solver. The boundary conditions for both the velocity and temperature as $r \rightarrow \infty$ are considered as finite values. For computation purpose, the coordinate $r$ (radial distance) is considered as varied from 1 to 2 where the value $r=2$ represents infinity. The convergence of the method is guaranteed by the satisfaction of the boundary conditions. The influence of various physical parameters such as the thermo-mechanical stress interaction coefficient $\left(a_{6}\right)$, strain thermal conductivity coefficient $\left(b_{3}\right)$, cross viscosity coefficient $\left(\mu_{c}\right)$ and Prandtl number $\left(p_{r}\right)$, on the velocity, temperature field and pressure distribution is shown in the form of tables and graphs. The numerical results presented in Tabs 1-3 show the solutions to highly non-linear coupled governing equations of velocity, temperature and pressure distribution with the various physical parameters.

Table 1. Numerical results of velocity field with various material parameters.

\begin{tabular}{|l|r|r|r|r|r|r|r|r|r|}
\hline \multirow{3}{*}{$\mathrm{R}$} & \multicolumn{6}{|c|}{ Velocity distribution $W(R)$ with $c=1, \rho=1, c 1=1, c 2=1, \mu=1, \mu_{c}=1, \eta_{a}=1, p_{a}=1, w_{a}=1, p_{r}=1$} \\
\cline { 2 - 12 } & \multicolumn{3}{|c|}{$b_{3}=1$} & \multicolumn{3}{|c|}{$b_{3}=3$} & \multicolumn{4}{c|}{$b_{3}=5$} \\
\cline { 2 - 12 } & $a_{6}=1$ & $a_{6}=2$ & $a_{6}=3$ & $a_{6}=1$ & $a_{6}=2$ & $a_{6}=3$ & $a_{6}=1$ & $a_{6}=2$ & $a_{6}=3$ \\
\hline 1 & 1 & 1 & 1 & 1 & 1 & 1 & 1 & 1 & 1 \\
\hline 1.1 & 1.520899 & 1.113976 & 1.004379 & 1.535279 & 1.303372 & 1.145115 & 1.540151 & 1.381759 & 1.253298 \\
\hline 1.2 & 1.988644 & 1.262008 & 1.019939 & 2.019922 & 1.623575 & 1.326993 & 2.030555 & 1.763813 & 1.534417 \\
\hline 1.3 & 2.417157 & 1.451608 & 1.055749 & 2.464818 & 1.96196 & 1.551572 & 2.481051 & 2.147631 & 1.844797 \\
\hline 1.4 & 2.817069 & 1.691822 & 1.126606 & 2.878359 & 2.320094 & 1.82551 & 2.899243 & 2.534623 & 2.186063 \\
\hline 1.5 & 3.19677 & 1.993568 & 1.256102 & 3.267178 & 2.699761 & 2.156295 & 3.291147 & 2.926167 & 2.56004 \\
\hline 1.6 & 3.5631 & 2.370057 & 1.481134 & 3.636637 & 3.102968 & 2.552367 & 3.661616 & 3.323621 & 2.968764 \\
\hline 1.7 & 3.921819 & 2.837303 & 1.858463 & 3.991165 & 3.53196 & 3.023259 & 4.01464 & 3.728345 & 3.414492 \\
\hline 1.8 & 4.277934 & 3.414749 & 2.474211 & 4.334496 & 3.989233 & 3.57975 & 4.353556 & 4.141712 & 3.899723 \\
\hline 1.9 & 4.63594 & 4.126047 & 3.457502 & 4.669838 & 4.477557 & 4.234049 & 4.681195 & 4.565119 & 4.427214 \\
\hline 2 & 5 & 5 & 5 & 5 & 5 & 5 & 5 & 5 & 5 \\
\hline
\end{tabular}

Table 2. Numerical results of temperature distribution with various material parameters.

\begin{tabular}{|l|r|r|r|r|r|r|r|r|r|}
\hline \multirow{3}{*}{$\mathrm{R}$} & \multicolumn{9}{|c|}{ Temperature distribution $T(R)$ with $c=1, \rho=1, c 1=1, c 2=1, \mu=1, \mu_{c}=1, \eta_{a}=1, p_{a}=1, w_{a}=1, p_{r}=1$} \\
\cline { 2 - 12 } & \multicolumn{2}{|c|}{$b_{3}=1$} & \multicolumn{6}{c|}{$b_{3}=3$} & \multicolumn{4}{c|}{$b_{3}=5$} \\
\cline { 2 - 12 } & $a_{6}=1$ & $a_{6}=2$ & $a_{6}=3$ & $a_{6}=1$ & $a_{6}=2$ & $a_{6}=3$ & $a_{6}=1$ & $a_{6}=2$ & $a_{6}=3$ \\
\hline 1 & 1 & 1 & 1 & 1 & 1 & 1 & 1 & 1 & 1 \\
\hline 1.1 & 1.470272 & 1.306681 & 1.243159 & 1.484652 & 1.38295 & 1.290809 & 1.489523 & 1.401379 & 1.328263 \\
\hline 1.2 & 1.901368 & 1.613435 & 1.544661 & 1.932646 & 1.789764 & 1.665425 & 1.943279 & 1.794218 & 1.692264 \\
\hline 1.3 & 2.305774 & 1.927136 & 1.831554 & 2.353434 & 2.184141 & 2.028452 & 2.369667 & 2.182311 & 2.048289 \\
\hline 1.4 & 2.692999 & 2.25473 & 2.111691 & 2.754289 & 2.569085 & 2.383622 & 2.775173 & 2.568866 & 2.401871 \\
\hline 1.5 & 3.070548 & 2.603598 & 2.394938 & 3.140956 & 2.947101 & 2.734056 & 3.164925 & 2.956695 & 2.758095 \\
\hline 1.6 & 3.444546 & 2.981895 & 2.694728 & 3.518083 & 3.32033 & 3.082436 & 3.543062 & 3.348351 & 3.121823 \\
\hline 1.7 & 3.820168 & 3.398895 & 3.030201 & 3.889514 & 3.69064 & 3.431136 & 3.912989 & 3.746224 & 3.497864 \\
\hline 1.8 & 4.201937 & 3.86537 & 3.429204 & 4.258498 & 4.059697 & 3.78231 & 4.277558 & 4.152611 & 3.891114 \\
\hline 1.9 & 4.593941 & 4.394022 & 3.932539 & 4.627838 & 4.429016 & 4.137963 & 4.639195 & 4.569777 & 4.306681 \\
\hline 2 & 5 & 5 & 5 & 5 & 5 & 5 & 5 & 5 & 5 \\
\hline
\end{tabular}


Table 3. Numerical results of pressure distribution with various material parameters.

\begin{tabular}{|c|c|c|c|c|c|c|c|c|c|c|c|c|}
\hline & \multicolumn{6}{|c|}{$\begin{array}{c}\text { Pressure distribution } P(R) \text { with } c=1, \rho=1, c 1=1, c 2=1 \text {, } \\
\mu=1, \mu_{c}=1, \eta_{a}=1, p_{a}=1, w_{a}=1, p_{r}=1 \quad b_{3}=1\end{array}$} & \multicolumn{6}{|c|}{$\begin{array}{c}\text { Pressure distribution } P(R) \text { with } c=1, \rho=1, c 1=1, c 2=1, \\
\mu=1, \mu_{c}=1, \eta_{a}=1, p_{a}=1, w_{a}=1, \mu_{c}=1, b_{3}=1\end{array}$} \\
\hline & \multicolumn{3}{|c|}{$a_{6}=1$} & \multicolumn{3}{|c|}{$a_{6}=2$} & \multicolumn{3}{|c|}{$a_{6}=1$} & \multicolumn{3}{|c|}{$a_{6}=2$} \\
\hline & $\mu_{c}=1$ & $\mu_{c}=2$ & $\mu_{c}=3$ & $\mu_{c}=1$ & $\mu_{c}=2$ & $\mu_{c}=3$ & $p_{r}=1$ & $p_{r}=2$ & $p_{r}=3$ & $p_{r}=1$ & $p_{r}=2$ & $p_{r}=3$ \\
\hline 1 & 1 & & & & & & & & & & & \\
\hline 1.1 & 954 & & 6376 & 03470 & & 690501 & & & 4.11997 & & -0.10358 & -0.14444 \\
\hline & & & & & & & & & & & & \\
\hline 1.3 & 6 & & & & & & & & & & & 5017 \\
\hline 4 & 50 & & & & & & & & & & & \\
\hline .5 & 175 & 36 & 08 & 4.7405 & 16.75 & 77068 & 17 & & -10.8 & 1 & 3.906 & 09901 \\
\hline 6 & .54 & & & & & & & & & & & 9.62093 \\
\hline .7 & 508 & 107 & & 18.605 & & & & & 1043 & 1866 & 20814 & 21.30836 \\
\hline & 5.11 & & 87 & & & 123.6651 & & & -9.55 & & & 72.33074 \\
\hline .9 & & & & & & & & & & & & 79.18548 \\
\hline 2 & -15.5403 & 20.6752 & -25.8101 & 84.3785 & 189.265 & 294.1515 & -15.5403 & 9.41701 & 6.27975 & 84.3785 & 123.6547 & 142.782 \\
\hline
\end{tabular}

\section{Special case for slow steady motion of the fluid}

\subsection{Governing equations}

The motion is assumed to be slow such that the non-linear terms in the equation of momentum and energy could be neglected. The equation of momentum and energy now reduce to the following:

The equation of momentum in the axial direction

$$
-C_{1}=\mu \nabla^{2} W-a_{6} C_{2} \nabla^{2} T
$$

and the energy equation

$$
\rho c C_{2} W=k \nabla^{2} T+b_{3} C_{2} \nabla^{2} W
$$

Equations (4.1) and (4.2) are coupled in terms of the velocity $W(R)$ and the temperature $T(R)$. In these two equations $C_{1}$ and $C_{2}$ represent constant pressure and temperature gradients, respectively. $a_{6}$ is the thermo-mechanical stress viscosity coefficient and $b_{3}$ is the strain thermal conductivity coefficient.

The appropriate boundary conditions for the velocity and temperature are

$$
W(R=1)=W_{a}, \quad W(R=\infty)=\text { finite, } \quad T(R=1)=1 \text { and } T(R=\infty)=\text { finite } .
$$

\subsection{Analytical and numerical solutions}

According to Anuradha [1] et al. analytical solutions to the coupled linear Eqs (4.1) and (4.2) together with the boundary conditions (4.3) yield the velocity distribution 


$$
W(R)=\frac{K_{0}(m R)}{K_{0}(m)} W_{a}+\frac{d_{1}}{m^{2}}\left\{1-\frac{K_{0}(m R)}{K_{0}(m)}\right\}
$$

and the temperature field

$$
T(R)=\frac{m_{1}}{m^{2}}\left\{\frac{K_{0}(m R)}{K_{0}(m)}-1\right\} W_{a}-\frac{d_{1} m_{1}}{m^{4}}\left\{\frac{K_{0}(m R)}{K_{0}(m)}-1\right\}+\frac{m_{1}}{4}\left\{\frac{d_{1}}{m^{2}}+b_{3}\right\}\left(R^{2}-1\right)+1
$$

where $K_{0}()$ is the modified Bessel function of order zero.

Here

$$
\begin{aligned}
& d_{1}=\frac{1}{1+a_{6} b_{3} p_{r}}, \quad m^{2}=\frac{a_{6} p_{r}}{1+a_{6} b_{3} p_{r}}, \quad m_{1}=\frac{d_{2} p_{r}}{1+a_{6} b_{3} p_{r}}, \quad d_{2}=\frac{\rho c_{1} c_{2} a^{4}}{\mu^{2} \eta_{a}}, \\
& a_{6}=\frac{\alpha_{6} \rho a^{2}}{\mu^{2}} c_{2}^{2}, \quad b_{3}=\frac{\beta_{3}}{\rho c a^{2}}, \quad p_{r}=\frac{\mu c}{k} .
\end{aligned}
$$

In order to get the analytical results of Eqs (4.4) and (4.5) for the velocity $W(R)$ and the temperature $T(R)$ for various values of physical parameters, the code of the algorithm has been executed in MATLAB running on a PC. The results are presented in Tabs 4-5.

In the present study, the numerical solutions of Eqs (4.1) and (4.2) with respect to the boundary conditions (4.3) are obtained numerically using the MATHEMATICA software package. An excellent convergence was achieved for all the results for slow steady motion of the fluid when compared to the analytical results. The numerical and analytical results for the velocity and temperature distributions are presented in Tabs 4-5. For computation purpose, the boundary conditions as $r \rightarrow \infty$ for the finite value are replaced by the value 5, the coordinate $r$ (radial distance) is considered as ranging from 1 to 2 where the value $r=2$ represents infinity. The numerical results obtained are compared with the analytical results of Anuradha[1] et.al and are found to be good in agreement. The analytical results obtained substantiate the validity and accuracy of the present numerical results.

Table 4. Comparison of velocity profiles for various material parameters.

\begin{tabular}{|c|c|c|c|c|c|c|c|c|}
\hline \multirow{2}{*}{$\mathrm{R}$} & \multicolumn{2}{|c|}{$\begin{array}{c}\text { Present Results for } \\
b_{3}=1\end{array}$} & \multicolumn{2}{c|}{$\begin{array}{c}\text { Results of Anuradha } \\
\text { for } b_{3}=1\end{array}$} & \multicolumn{2}{c|}{$\begin{array}{c}\text { Present Results for } \\
b_{3}=3\end{array}$} & \multicolumn{2}{c|}{$\begin{array}{c}\text { Results of Anuradha } \\
\text { for } b_{3}=3\end{array}$} \\
\cline { 2 - 9 } & $a_{6}=1$ & $a_{6}=2$ & $a_{6}=1$ & $a_{6}=2$ & $a_{6}=1$ & $a_{6}=2$ & $a_{6}=1$ & $a_{6}=2$ \\
\hline 1 & 1 & 1 & 1 & 1 & 1 & 1 & 1 & 1 \\
\hline 1.1 & 1.506824 & 1.477614 & 1.506810 & 1.477590 & 1.527799 & 1.517688 & 1.527791 & 1.517699 \\
\hline 1.2 & 1.971945 & 1.919882 & 1.971951 & 1.919862 & 2.010905 & 1.993084 & 2.010890 & 1.993076 \\
\hline 1.3 & 2.404484 & 2.335827 & 2.404495 & 2.335805 & 2.457747 & 2.434504 & 2.457748 & 2.434493 \\
\hline 1.4 & 2.811721 & 2.732729 & 2.811731 & 2.732739 & 2.87497 & 2.848521 & 2.87495 & 2.848500 \\
\hline 1.5 & 3.199603 & 3.116622 & 3.199585 & 3.116600 & 3.267924 & 3.240445 & 3.267900 & 3.240428 \\
\hline 1.6 & 3.573094 & 3.492626 & 3.573076 & 3.492636 & 3.640998 & 3.614647 & 3.640979 & 3.614635 \\
\hline 1.7 & 3.936419 & 3.86519 & 3.936400 & 3.865100 & 3.997852 & 3.974790 & 3.997851 & 3.974791 \\
\hline 1.8 & 4.293244 & 4.238265 & 4.293222 & 4.238274 & 4.341588 & 4.32399 & 4.341569 & 4.32376 \\
\hline 1.9 & 4.646803 & 4.615431 & 4.646793 & 4.615422 & 4.674866 & 4.664941 & 4.674865 & 4.664922 \\
\hline 2 & 5 & 5 & 5 & 5 & 5 & 5 & 5 & 5 \\
\hline
\end{tabular}


Table 5. Comparison of temperature profiles for various material parameters.

\begin{tabular}{|c|c|c|c|c|c|c|c|c|}
\hline \multirow{2}{*}{ R } & \multicolumn{2}{|c|}{$\begin{array}{c}\text { Present Results for } \\
b_{3}=1\end{array}$} & $\begin{array}{c}\text { Results of Anuradha for } \\
b_{3}=1\end{array}$ & \multicolumn{2}{c|}{$\begin{array}{c}\text { Present Results for } \\
b_{3}=3\end{array}$} & \multicolumn{2}{c|}{$\begin{array}{c}\text { Results of Anuradha For } \\
b_{3}=3\end{array}$} \\
\cline { 2 - 9 } & $a_{6}=1$ & $a_{6}=2$ & $a_{6}=1$ & $a_{6}=2$ & $a_{6}=1$ & $a_{6}=2$ & $a_{6}=1$ & \\
\hline 1 & 1 & 1 & 1 & 1 & 1 & 1 & 1 & 1 \\
\hline 1.1 & 1.456196 & 1.48850 & 1.456177 & 1.488761 & 1.477171 & 1.508539 & 1.477190 & 1.508539 \\
\hline 1.2 & 1.884669 & 1.942372 & 1.884681 & 1.942382 & 1.923629 & 1.978976 & 1.923630 & 1.978970 \\
\hline 1.3 & 2.29310 & 2.369245 & 2.29291 & 2.369235 & 2.346363 & 2.418584 & 2.346333 & 2.418588 \\
\hline 1.4 & 2.687651 & 2.775183 & 2.687662 & 2.775164 & 2.75090 & 2.833082 & 2.75092 & 2.833069 \\
\hline 1.5 & 3.073381 & 3.165125 & 3.073393 & 3.165111 & 3.141702 & 3.227037 & 3.141700 & 3.227034 \\
\hline 1.6 & 3.454540 & 3.543180 & 3.454522 & 3.543171 & 3.522444 & 3.604380 & 3.522445 & 3.604389 \\
\hline 1.7 & 3.834768 & 3.912839 & 3.834749 & 3.912850 & 3.896201 & 3.967650 & 3.896208 & 3.967639 \\
\hline 1.8 & 4.217246 & 4.277127 & 4.217245 & 4.277108 & 4.26559 & 4.31999 & 4.26567 & 4.31998 \\
\hline 1.9 & 4.604803 & 4.638715 & 4.604784 & 4.638693 & 4.632866 & 4.66347 & 4.632860 & 4.66350 \\
\hline 2 & 5 & 5 & 5 & 5 & 5 & 5 & 5 & 5 \\
\hline
\end{tabular}

\section{Results analysis and discussion}

The results are illustrated graphically in Figs 2-14. To get the physical insight into the problem the velocity, temperature field, pressure distribution have been discussed by assigning numerical values to various material parameters such as the thermo-mechanical interaction coefficient $\left(a_{6}\right)$, the strain thermal conductivity coefficient $\left(b_{3}\right)$, cross viscosity coefficient $\left(\mu_{c}\right)$ and Prandtl number $\left(p_{r}\right)$ which characterise the flow phenomena. The influences of these parameters on the velocity, temperature and pressure distribution have been studied and are presented graphically.

\subsection{Velocity field}

The impact variation of the thermo-mechanical stress interaction coefficient $\left(a_{6}\right)$ on the velocity field is presented graphically in Figs 2-4 and the influence of the strain thermal conductivity coefficient $\left(b_{3}\right)$ on the velocity field is shown in Fig.5. Figures 2-4 show that the velocity of the fluid decreases as the values of the thermo-mechanical stress interaction coefficient $\left(a_{6}\right)$ increase and the velocity of the fluid increases with the increase of the distance from the pipe and attains maximum velocity far away from the tube boundary for $R>>1$. It is observed from Fig. 5 that the velocity of the fluid increases at a slower rate for an increasing value of the thermal conductivity coefficient $\left(b_{3}\right)$. This signifies that the effect of the thermal conductivity coefficient on the flow field is much smaller. 


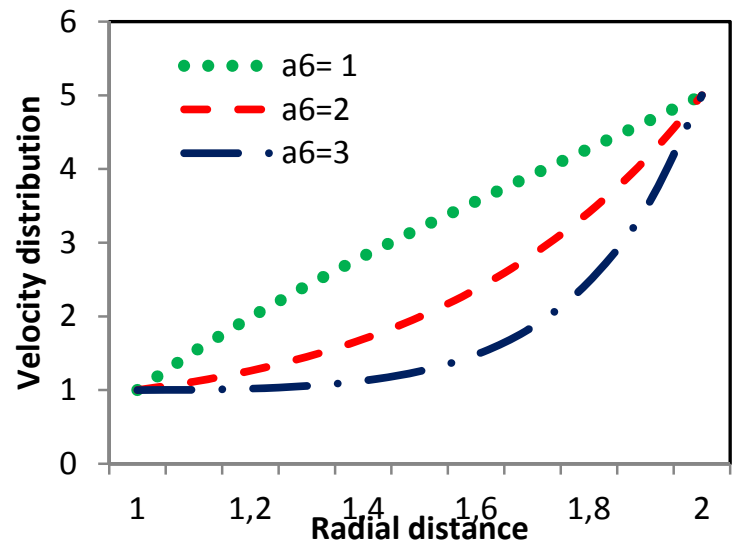

Fig.2. Variation of velocity for $b_{3}=1$.

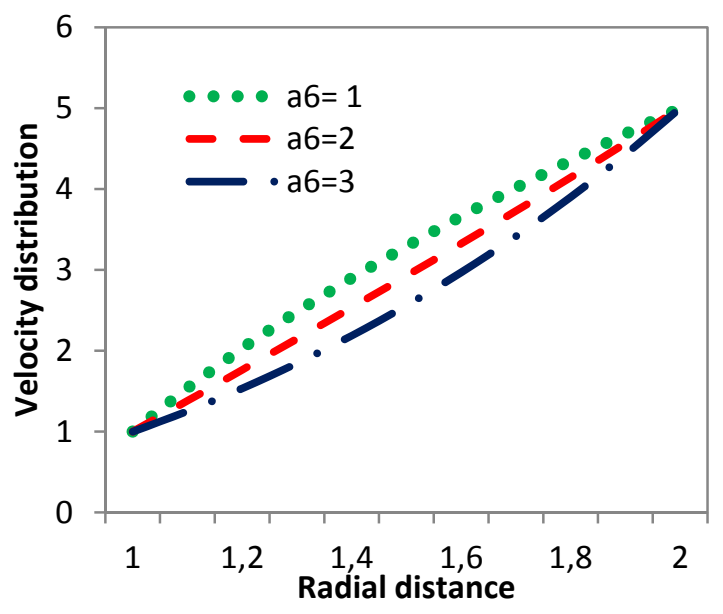

Fig.4. Variation of velocity for $b_{3}=5$.

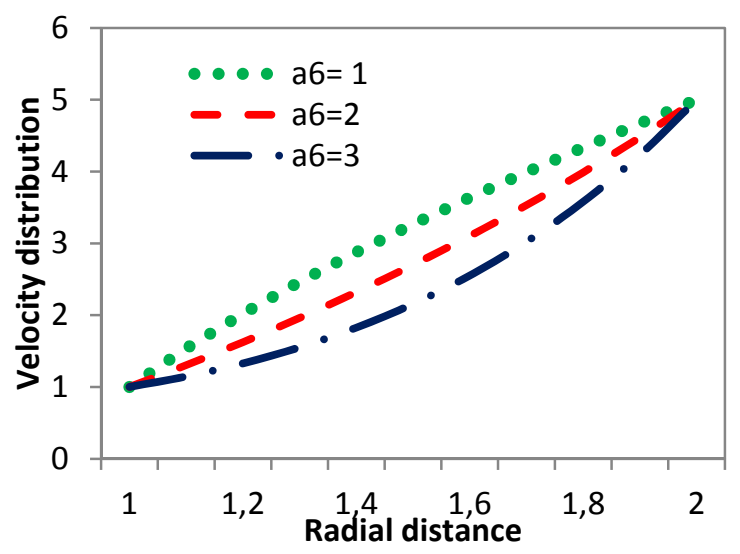

Fig.3. Variation of velocity for $b_{3}=3$.

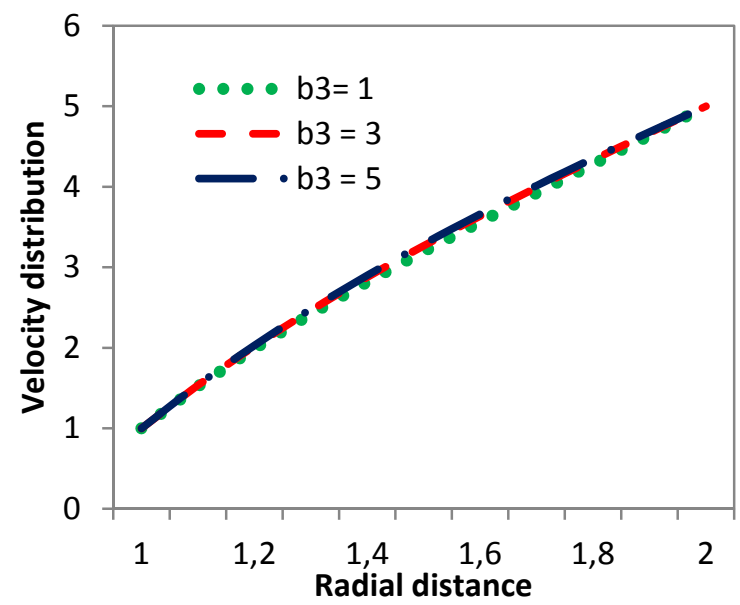

Fig.5. Variation of velocity for $a_{6}=1$.

\subsection{Temperature distribution}

It is observed that the material parameter thermo-mechanical stress interaction coefficient $\left(a_{6}\right)$ is influenced by the temperature distribution and which is graphically shown in Figs 6-8. The effect of the strain thermal conductivity coefficient $\left(b_{3}\right)$ is depicted in Fig.9. It is noticed that the temperature distribution decreases with the increasing values of thermo-mechanical stress interaction coefficient $\left(a_{6}\right)$. This is due to the greater conversion of thermal energy to kinetic energy. The reverse effect is observed in the variation of temperature distribution for different values of the strain thermal conductivity coefficient $\left(b_{3}\right)$. From Fig.9, it is observed that the rate of increase of temperature distribution is very slow and all the temperature profiles coincide far away from the tube boundary. 


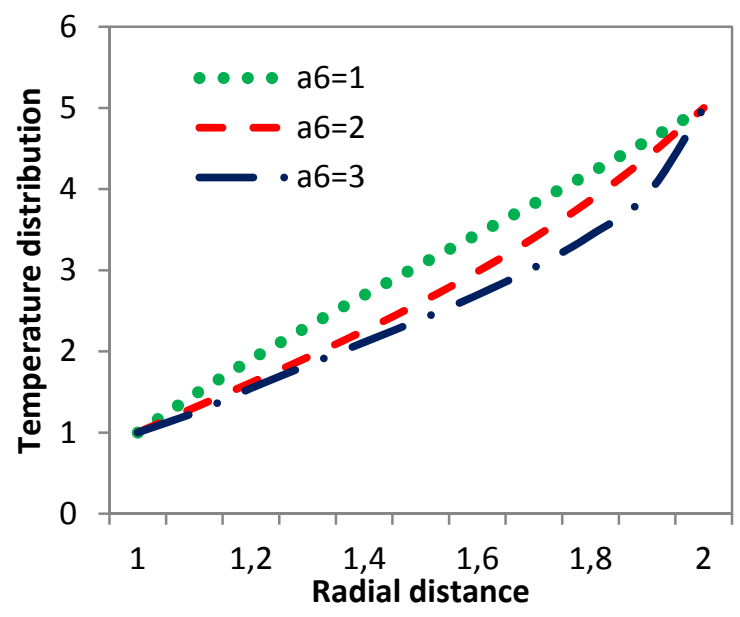

Fig.6. Variation of temperature for $b_{3}=1$.

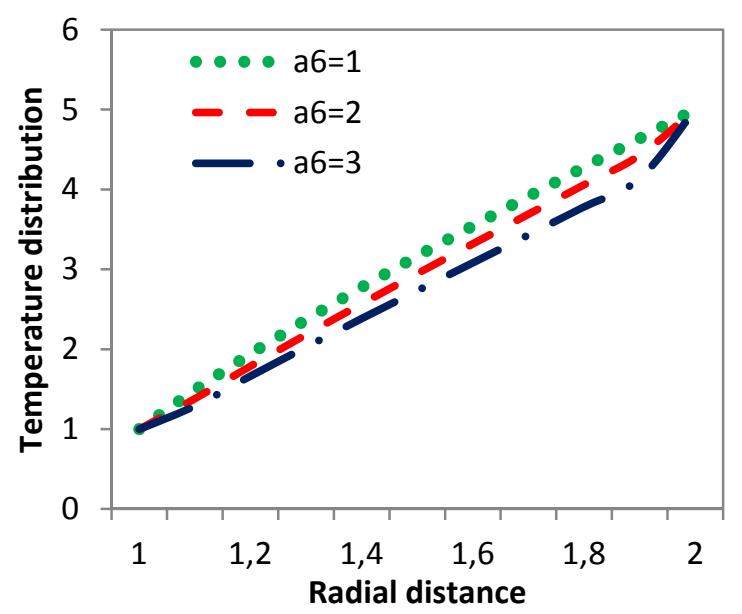

Fig.8. Variation of temperature for $b_{3}=5$.

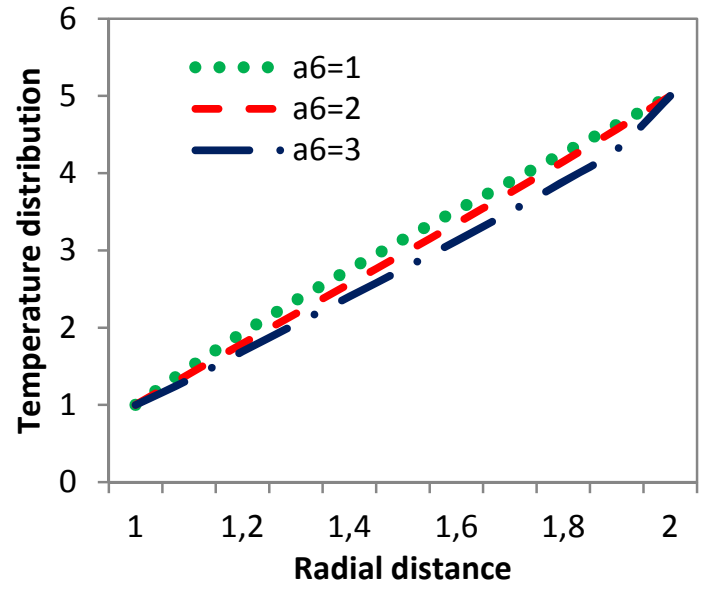

Fig.7. Variation of temperature for $b_{3}=3$.

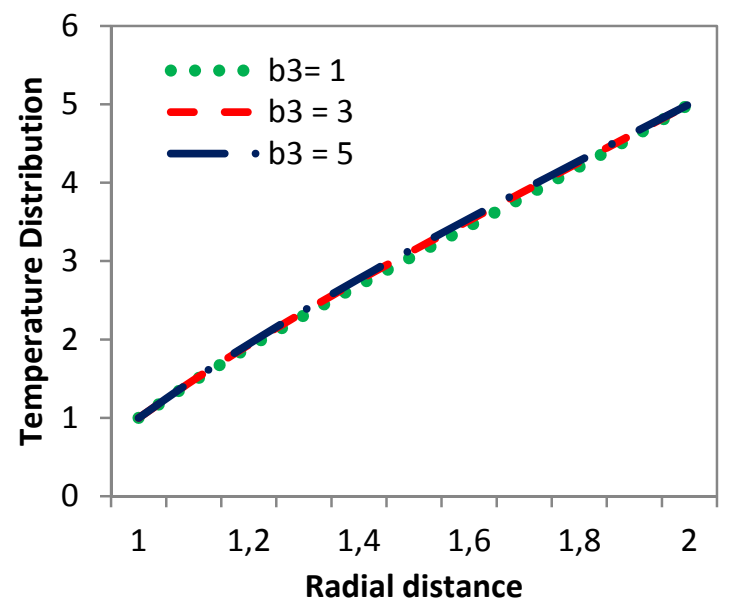

Fig.9. Variation of temperature or $a_{6}=1$.

\subsection{Pressure distribution}

The pressure distribution throughout the boundary is in the direction normal to the cylindrical surface and which is influenced by the Reiner Rivlin cross viscosity coefficient $\left(\mu_{c}\right)$. The effects of the Prandtl number $\left(p_{r}\right)$ and cross viscosity coefficient $\left(\mu_{c}\right)$ on the pressure distribution are presented in Figs 10-13. From Fig.10, it can be noted that the pressure distribution decreases with the increase of the cross viscosity coefficient $\left(\mu_{c}\right)$ whereas Fig. 11 shows that the flow suddenly takes a reverse direction to the positive side and increases with the increase of the cross viscosity coefficient $\left(\mu_{c}\right)$. This is due to the effect of the thermo-mechanical stress interaction coefficient of thermo viscous fluid flows. It is interesting to note that there is a marked departure between the flows of thermo-viscous fluids when compared to that of Newtonian fluids. The dimensionless parameter Prandtl number is the ratio between the kinematic viscosity and the thermal diffusivity, the effect of this on the pressure distribution is shown in Figs 12-13. Figure 12 presents that the pressure distribution increases with the increase of the Prandtl number $\left(p_{r}\right)$ and suddenly takes in to the positive direction. This is also due to the influence of the thermo-mechanical stress interaction coefficient $\left(a_{6}\right)$. The physical influence of this in heat transfer problems is the Prandtl number controls the thickness of the velocity and thermal boundary layers. 

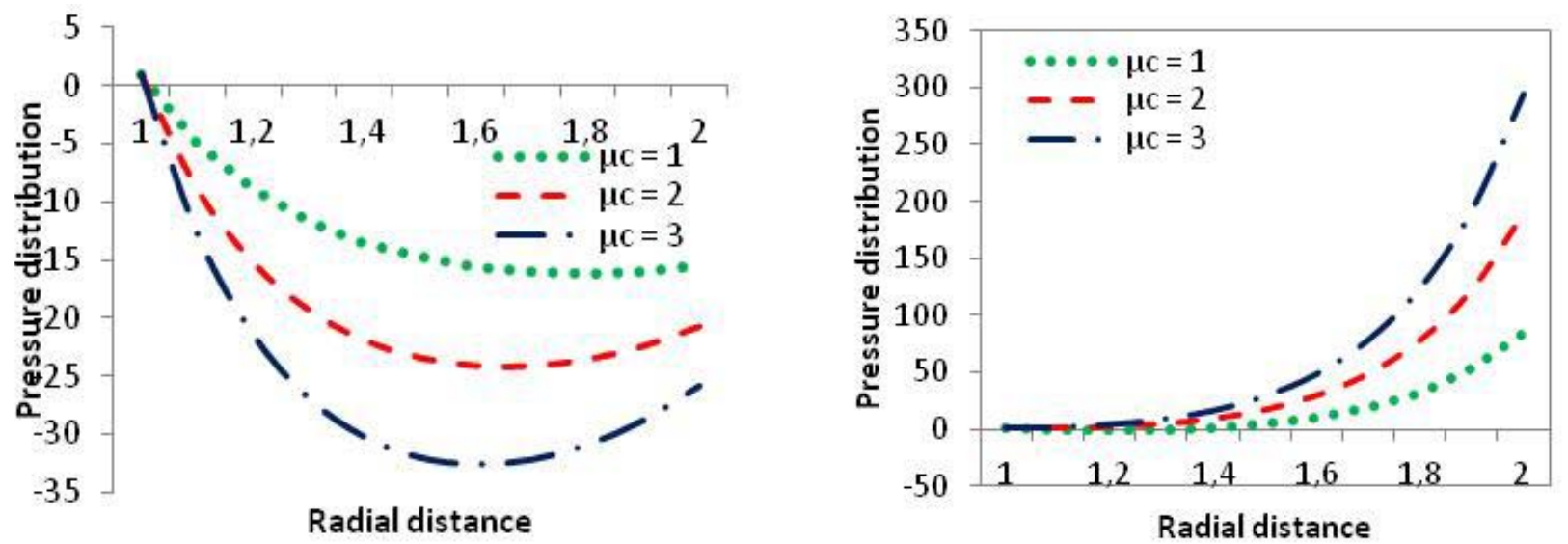

Fig.10. Variation of pressure distribution for $a_{6}=1$. Fig.11. Variation of pressure distribution for $a_{6}=2$.
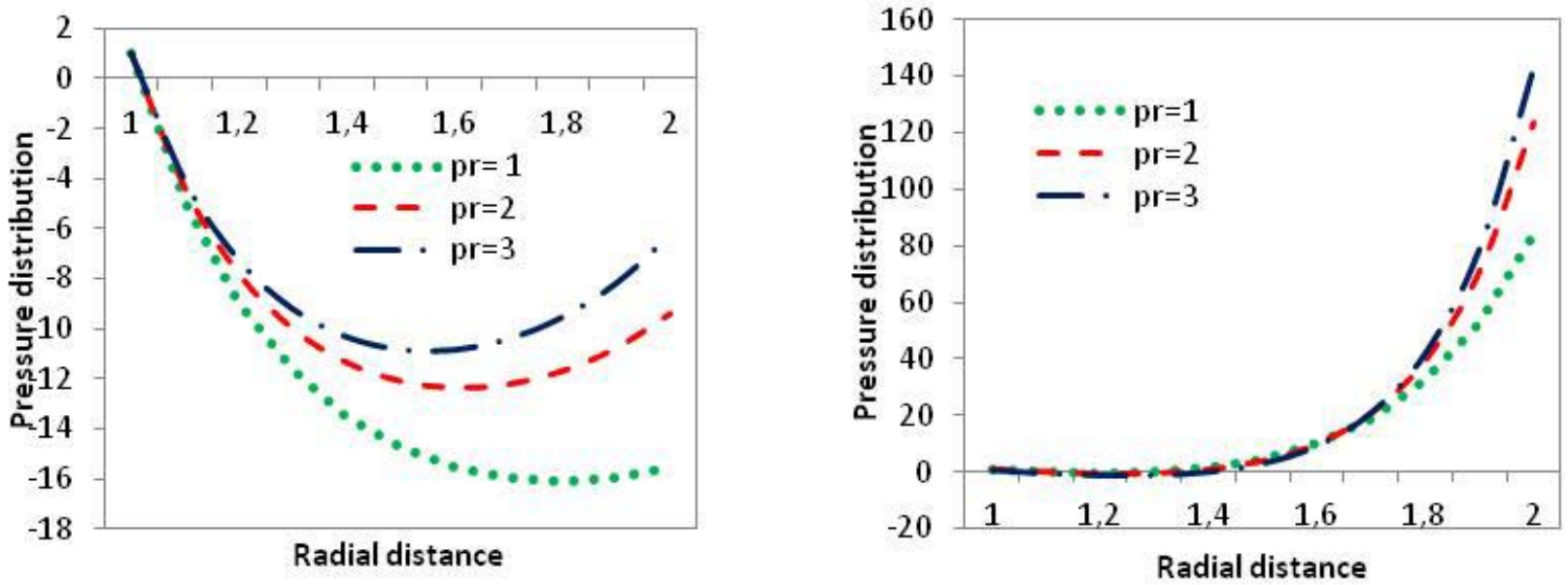

Fig.12. Variation of pressure distribution for $a_{6}=1$.

Fig.13. Variation of pressure distribution for $a_{6}=2$.

(a)

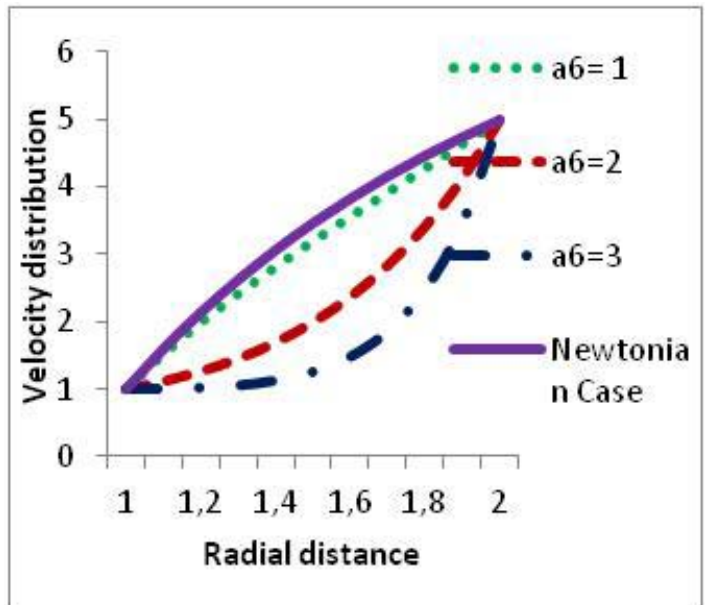

(b)

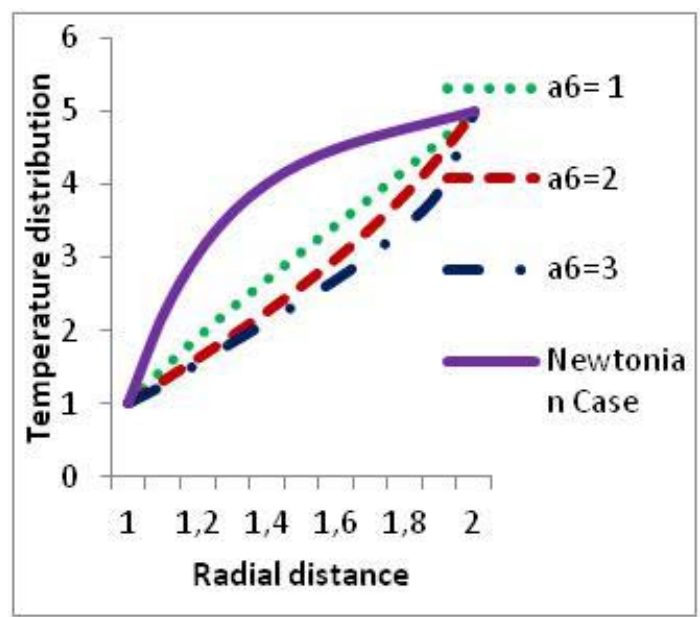

Fig.14. Comparison of (a) velocity and (b) temperature for a Newtonian fluid. 


\subsection{Comparison with a Newtonian case}

To make a comparative study, in Figs $14 \mathrm{a}$ and $\mathrm{b}$ the variation of the velocity and temperature for a Newtonian-viscous fluid is presented graphically. For a Newtonian fluid the parabolic profile is realized for both the velocity and the temperature distributions. It can be noticed that the velocity of the thermo-viscous fluid is smaller compared to that of a classical Newtonian-viscous fluid, while the temperature of the thermoviscous fluid is much less compared to that of classical Newtonian-viscous fluid. It is observed that the temperature profiles of thermo-viscous fluids deviate very much from the classical viscous fluids. This is due to the fact that the decoupled nature of velocity and temperature of the fluid in momentum and energy equations. Therefore the interaction between thermal and mechanical responses of fluids in motion are lost in Newtonian type of fluids.

\section{CONCLUSIONS}

In this paper, the steady thermo-viscous incompressible fluid flows around a circular cylinder are studied numerically. The resulting governing steady, non-linear and coupled equations are solved by using MATHEMATICA software ND solver. The computations are carried out for different values of $a_{6}, b_{3}, \mu_{c}$, $p_{r}$ and for the fixed values of other physical parameters.

1. The fluid velocity decreases with the increase of the thermo-mechanical stress interaction coefficient $\left(a_{6}\right)$ while it increases as the thermal conductivity coefficient $\left(b_{3}\right)$ increases.

2. The fluid temperature decreases with the increase of the thermo-mechanical stress interaction coefficient $\left(a_{6}\right)$ while it increases as the strain thermal conductivity coefficient $\left(b_{3}\right)$ increases.

3. The fluid pressure decreases as increase in cross-viscosity coefficient $\left(\mu_{c}\right)$ while increases as thermomechanical stress interaction coefficient $\left(a_{6}\right)$ increases.

4. The fluid pressure increases as increase in Prandtl number $\left(p_{r}\right)$ and thermo-mechanical stress interaction coefficient $\left(a_{6}\right)$.

5. The numerical results obtained using MATHEMATICA ND solver are in good agreement with the analytical results obtained in terms of modified Bessel functions.

\section{Nomenclature}

$a_{6}-$ dimensionless thermo mechanical stress interaction coefficient

$b_{i j}$ - thermal gradient bivector

$b_{3}-$ dimensionless strain thermal conductivity coefficient

$C_{1}$ - constant pressure gradient

$C_{2}$ - constant temperature gradient

$c$ - specific heat

$d_{i j}$ - rate of deformation tensor

$f_{i}-i^{\text {th }}$ component of external force per unit mass

$p_{r} \quad-$ Prandtl number

$R$ - dimensionless radial distance

$r$ - radial distance

$T$ - dimensionless temperature

$t_{j i}-$ stress tensor

$u_{i}-i^{\text {th }}$ component of velocity 


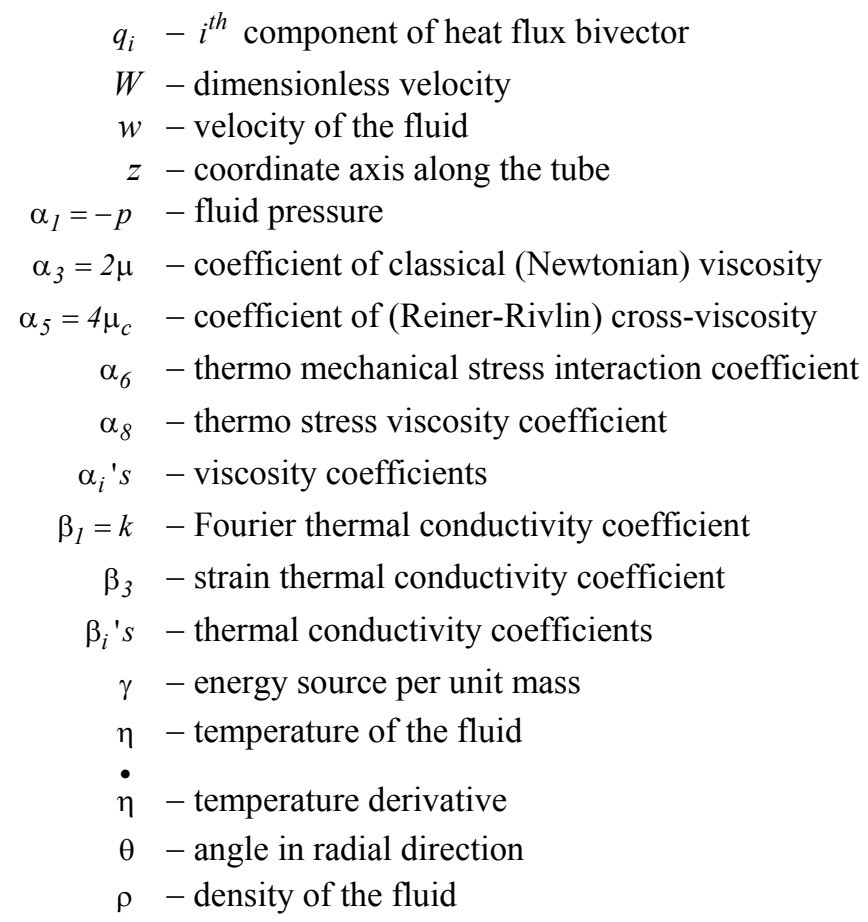

\section{References}

[1] Anuradha K. (2006): On steady and unsteady flows of thermo-viscous fluids. - Ph. D Thesis.- J.N.T.U. Hyderabad, India.

[2] Green A.E. and Naghdi P.M. (1965): A dynamical theory of interacting continua. - Int. J. Engg. Sci., vol.3, pp.231-241.

[3] Green A.E. and Naghdi P.M. (1995): A new thermo-viscous theory for fluids. - Journal of Non-Newtonian Fluid Mechanics, vol.56, No.3, pp.289-306.

[4] Kelly P.D. (1965): Some viscometric flows of incompressible thermo-viscous fluids. - Int. J. Engg. Sci., vol.2, pp.519-537.

[5] Koh S.L. and Eringin A.C. (1963): On the foundations of non-linear thermo-elastic fluids. - Int. J. Engg. Sci., vol.1, pp.199-229.

[6] Langlois W.E. and Rivlin R.S. (1963): Slow steady flow of viscoelastic fluids through non-linear tubes. Rendiconti di Mathematica, vol.22, pp.169-175.

[7] Muthuraj R. and Srinivas S. (2007): Flow of a thermo-viscous fluid through an annular tube with constriction. Defence Science Journal, vol.57, No.5, pp.653-659.

[8] Md. Hossain M., Mandal A.C., Roy N.C. and Hossain M.A. (2013): Flow of thermomicropolar fluid past a vertical surface. - Applications and AppliedMathematics: An International Journal, vol.8, No.1, pp.128-150.

[9] Nagaratnam E. (2006): Some steady and unsteady flows of thermo-viscous fluids. - Ph. D Thesis, J.N.T.U Hyderabad, India.

[10] Nageswara Rao P. (1979): Some problems in thermo-viscous fluid dynamics. - Ph. D Thesis, K.U. Warangal, India.

[11] Nageswara Rao P. and Pattabhi Ramacharyulu N.Ch. (1979): Steady flow of a second order thermo- viscous fluid over an infinite plate. - Proc. Ind. Acad. Sci., vol.88A, Part III No.2, pp.157-162. 
[12] Nageswara Rao P. and Pattabhi Ramacharyulu N.Ch. (1979): Steady flow of a thermo-viscous fluid through straight tubes. - Journal of Ind. Inst. Sci., vol.61(B), pp.89-102.

[13] Nageswara Rao P. and Pattabhi Ramacharyulu N.Ch. (1980): A note on steady slow motion of thermo-viscous fluid through a circular tube. - Indian Journal of Pure and Appl. Math., vol.11, No.4, pp.487-491.

[14] Pattabhi Ramacharyulu N.Ch. (1966): Problems in the steady of non-Newtonian fluid dynamics. - Ph. D Thesis, O.U. Hyderabad, India.

[15] Pothanna N., Nageswara Rao P. and Pattabhi Ramacharyulu N.Ch. (2015): Flow of slightly thermo-viscous fluid in a porous slab bounded between two permeable parallel plates. - Int. J. Adv. Appl. Math. and Mech., vol.2, No.3, pp.1-9.

[16] Pothanna N., Srinivas J., Nageswara rao P. and Pattabhi Ramacharyulu N.Ch. (2014): Linearization of thermoviscous fluid in a porous slab bounded between two fixed permeable horizontal parallel plates in the absence of thermo-mechanical interaction coefficient. - International Journal of Modern Trends in Engineering and Research, vol.1, No.5, pp.412-424.

[17] Pothanna N., Pattabhi Ramacharyulu N.Ch. and Nageswara Rao P. (2015): Effect of strain thermal conductivity on slightly thermo-viscous fluid in a porous slab bounded between two parallel plates. - Journal of Advanced Computing, vol.4, No.1, pp.37-58.

[18] Pothanna N., Aparna P. and Srinivas J. (2016): Unsteady forced oscillations of a fluid bounded by rigid bottom. - International Journal of Control Theory and Applications vol.9, No.19, pp.9049-9054.

[19] Srinivas J., Pothanna N., Nageswara Rao P. and Pattabhi Ramacharyulu N.Ch. (2013): Slow steady motion of a thermo-viscous fluid between two parallel plates with constant pressure and temperature gradients. International Journal of Research in Engineering and Technology, vol.2, No.11, pp.294-299.

[20] Srinivas S. and Muthuraj R. (2005): Flow of a thermo-viscous fluid in a radially non-symmetric constricted tube. - Journal of Ultra Scientist of Physical sciences, vol.17, pp.437-442.

[21] Srinivas S., Srinivas Ch. V. and Pattabhi Ramacharyulu N.Ch. (1995): Peristaltic transport of a thermo-viscous fluid. - Acta Ciencia Indica, vol.47, pp.1157-1167.

Received: January 18, 2017

Revised: March 21, 2017 